\title{
THE TWO-GENERATOR SUBGROUPS OF ONE-RELATOR GROUPS WITH TORSION
}

BY

\author{
STEPHEN J. PRIDE
}

\begin{abstract}
The main aim of this paper is to show that every two-generator subgroup of any one-relator group with torsion is either a free product of cyclic groups or is a one-relator group with torsion. This result is proved by using techniques for reducing pairs of elements in certain $H N N$ groups. These techniques not only apply to one-relator groups with torsion but also to a large number of other groups, and some additional applications of the techniques are included in the paper. In particular, examples are given to show that the following result of $\mathrm{K}$. Honda is no longer true for infinite groups: if $\boldsymbol{g}$ is a commutator in a finite group $\boldsymbol{G}$ then every generator of $\operatorname{sgp}\{g\}$ is a commutator in $G$. This confirms a conjecture of B. H. Neumann.
\end{abstract}

1. Introduction. The principal aim of this paper is to establish the following result.

MAIN TheOREM. Let $G=\left\langle t, a, b, \ldots ; R^{n}\right\rangle$, where $R$ is cyclically reduced and $n>1$. Every two-generator subgroup of $G$ is either a free product of two cyclic groups or is a one-relator group with torsion.

This theorem will be proved using results and techniques developed in [5], [6] and, most especially, in [7]. Indeed this paper should be regarded as an addendum to [7]. The book [3] by Magnus, Karrass and Solitar will be used throughout as standard reference for notation and background material. Unexplained concepts and notation which cannot be found in [3] will be as in [5], [6] and [7]. Complete familiarity with $\$ 2$ of [7] will be assumed.

An immediate consequence of the Main Theorem is: every two-generator subgroup of a one-relator group with torsion is either free or has torsion. This reinforces the feeling many people have that, apart from the complications introduced by having elements of finite order, one-relator groups with torsion behave much like free groups. B. B. Newman and I once thought that every finitely generated subgroup of a one-relator group with torsion would either be free or have torsion. However, Newman has provided the following

Received by the editors May 10, 1976.

AMS (MOS) subject classifications (1970). Primary 20E10, 20E30, 20F05, 20F10; Secondary 20F35.

- American Mathematical Society 1977 
example to show that this need not be the case, even for three-generator subgroups.

Let $G=\left\langle a, t ;\left(t^{-1} a^{-1} t a^{2}\right)^{2}\right\rangle$ and let $L$ be the normal subgroup generated by $a^{2}, t$. Then, as a group, $L$ is generated by $t, a^{2}, a t a^{-1}$, and has presentation

$$
L=\left\langle t, y, z ;\left[y, z^{-1}\right]\left[t^{-1}, y^{-1}\right] y\right\rangle
$$

under the mapping $t \mapsto t, y \mapsto a^{2}, z \mapsto a t a^{-1}$. It can be shown that the relator of $L$ is not a primitive in the free group on $t, y, z$, so $L$ is not free.

Another consequence of the Main Theorem is the following result.

Let $G=\left\langle t, a, b, \ldots ; R^{n}\right\rangle$ where $\left.n\right\rangle 1$. Let $u, v$ be a pair of words in the generators of $G$. Then either every relation between $u$ and $v$ is a consequence of a single relation $T^{l}(u, v)=1$ with $l>1$, or there is a pair of associated primitives $P(x, y), Q(x, y)$ in the free group on $x, y$, and every relation between $u$ and $v$ is a consequence of relations $P^{p}(u, v)=1, Q^{q}(u, v)=1$ for certain integers $p, q$.

To prove this, note that, by the Main Theorem, there is a generating pair $(w, z)$ of $\operatorname{sgp}\{u, v\}$ such that either every relation between $w$ and $x$ is a consequence of relations $w^{p}=1, z^{q}=1$ for certain integers $p, q$, or every relation between $w$ and $z$ is a consequence of a single relation $S^{l}(w, z)=1$ with $l>1$. Now free products of two cyclic groups have one $T$-system of generating pairs by the Grushko-Neumann Theorem, and two-generator one-relator groups with torsion have one $T$-system of generating pairs by the Principal Lemma of [7]. Thus $(u, v)$ lies in the same $T$-system as $(w, z)$. Consequently (see [5, p. 333]), there is an automorphism $\psi$ of the free group on $x, y$ such that if $X_{1}(w, z)=1, \ldots, X_{r}(w, z)=1$ is a system of defining relations between the generators $w, z$, and if $\psi$ is applied to each of the words $X_{1}(x, y), \ldots, X_{r}(x, y)$ to get words $Y_{1}(x, y), \ldots, Y_{r}(x, y)$, then $Y_{1}(u, v)=$ $1, \ldots, Y_{r}(u, v)=1$ constitutes a system of defining relations between the generators $u, v$. The result now follows.

In actual fact the above result can be obtained directly from the proof of the Main Theorem. In addition, it can be shown that if $G$ is countably generated then there is an algorithm to obtain for any pair of words $u, v$ in the generators of $G$ a system of defining relations between $u$ and $v$ of the form described above. An explicit proof of this result will not be given, but the proof of the Main Theorem will be presented in such a way so as to enable the reader to deduce the result if he or she desires to do so. As well as this, some other (simpler) applications of the techniques used in the proof of the Main Theorem are given below, and reasonably detailed information concerning algorithmic questions is given there.

In order to prove the Main Theorem the following result is required. Recall 
[5] that if $G$ is an $H N N$ group with stable letter $t$, then the following transformations of pairs $\left(W_{1}, W_{2}\right)$ of $t$-reduced words are called elementary transformations:

(a) interchanging $W_{1}$ and $W_{2}$;

(b) replacing $W_{i}$ by $W_{i}^{i}$ for $i=1,2$;

(c) replacing $W_{i}$ by the $t$-reduced form of one of $W_{j}^{e} W_{i}, W_{i} W_{j}^{e}$ for $i \neq j$ and leaving $W_{j}$ fixed;

(d) replacing $W_{1}$ and $W_{2}$ by the $t$-reduced forms of $T^{-1} W_{1} T, T^{-1} W_{2} T$ respectively, where $T$ is a $t$-reduced word.

THEOREM 1. Let $G$ be an $H N N$ group with base $H$, stable letter $t$, and associated subgroups $K_{-1}$ and $K_{1}$. Suppose that $K_{-1}$ and $K_{1}$ are malnormal subgroups of $H$. Let $(X, Y)$ be a pair of $t$-reduced words in the generators of $G$. Then $(X, Y)$ can be transformed by a finite sequence of elementary transformations to a pair $(\hat{X}, \hat{Y})$ where either:

(1) $\operatorname{sgp}\{\hat{X}, \hat{Y}\}=\operatorname{sgp}\{\hat{X}\} * \operatorname{sgp}\{\hat{Y}\} ;$ or

(2) $\hat{X}$ and $\hat{Y}$ are $t$-free; or

(3) $\hat{X}$ has the form $t h_{1} t h_{2} \cdots t h_{r}$ where $r>0$ and the $h_{i}$ are $t$-free, $\hat{Y}$ is $t$-free and defines a nontrivial element of $K_{-1}, \hat{X}^{-1} \hat{Y} \hat{X} t$-reduces to a $t$-free word.

If $t$-reducing is algorithmic then there is an algorithm for obtaining for any pair $(X, Y)$ the pair $(\hat{X}, \hat{Y})$.

This result is a generalization of Theorem 6 of [5], and indeed the proof is a refinement of the proof of that result. The proof will be given in $\$ 2$.

The importance of Theorem 1 is that in many cases it provides a link between the two-generator subgroup structure of $G$ and the two-generator subgroup structure of the base $H$. This is particularly helpful when it comes to dealing with one-relator groups using the inductive techniques developed by Magnus and others. Theorem 1 is also helpful in analysing the two-generator subgroup structure of a large number of groups which are not one-relator groups. Partly to illustrate this, and partly to pave the way for the calculations needed to prove the Main Theorem, some additional applications of Theorem 1 are now given.

Let $H=\left\langle a, b ; R_{1}, R_{2}, \ldots\right\rangle$ and assume $a$ and $b$ have the same order. Assume further that $\operatorname{sgp}\{a\}$ and $\operatorname{sgp}\{b\}$ are malnormal in $H$, and that no nontrivial power of $a$ is conjugate to a power of $b .\left(^{1}\right)$ Let $G=$ $\left\langle a, b, t ; R_{1}, R_{2}, \ldots, t^{-1} a t=b\right\rangle$. It will be shown in certain circumstances that the two-generator subgroup structure of $G$ is completely determined by the two-generator subgroup structure of $H$. Indeed, assuming certain equations hold in $H$ (see (4) and (5)), it turns out that every two-generator subgroup of $G$ is isomorphic to either a free product of cyclic groups, or a subgroup of $H$, or an

(') Examples of groups satisfying these conditions can be found in $[5,8 \S 1.4,3.1,3.2,3.3]$. 
HNN group with base a two-generator subgroup $\operatorname{sgp}\left\{a^{m}, h^{-1} b^{m} h\right\}$ of $H$ and with associated subgroups $\operatorname{sgp}\left\{a^{m}\right\}, \operatorname{sgp}\left\{h^{-1} b^{m} h\right\} .\left(^{2}\right)$ In addition, suppose that for each pair of words $h_{1}, h_{2}$ in $a, b$ there is given a set $\mathbf{D}\left(h_{1}, h_{2}\right)$ of words in variables $x, y$ such that the kernel of the homomorphism $x \mapsto h_{1}, y \mapsto h_{2}$ of the free group on $x, y$ to $\operatorname{sgp}\left\{h_{1}, h_{2}\right\}$ is the normal closure of $\mathbf{D}\left(h_{1}, h_{2}\right)$-in other words, $\left\langle x, y ; W(x, y)\left(W \in \mathbf{D}\left(h_{1}, h_{2}\right)\right)\right\rangle$ is a presentation for $\operatorname{sgp}\left\{h_{1}, h_{2}\right\}$ associated with the generators $h_{1}, h_{2}$. Then using these given presentations of the two-generator subgroups of $H$ there is a method for obtaining for any pair of t-reduced words $(X, Y)$ in the generators of $G$ a presentation for $\operatorname{sgp}\{X, Y\}$ associated with the generators $X, Y$. This method is algorithmic provided the following conditions are satisfied: the generalised word problem for $\operatorname{sgp}\{a\}$ and $\operatorname{sgp}\{b\}$ in $H$ is solvable, so that $t$-reducing is algorithmic; there is an algorithm to calculate for any word $h$ in $a$ and $b$ the order in $H$ of the element defined by $h$ (i.e. the order problem for $H$ is solvable).

Let $(X, Y)$ be a pair of $t$-reduced words in the generators of $G$, and obtain from $(X, Y)$ the pair $(\hat{X}, \hat{Y})$ as in Theorem 1 . Then there is a finite sequence of pairs $\left(X_{0}, Y_{0}\right),\left(X_{1}, Y_{1}\right), \ldots,\left(X_{l}, Y_{l}\right)$ where $\left(X_{0}, Y_{0}\right)=(X, Y),\left(X_{l}, Y_{l}\right)=$ $(\hat{X}, \hat{Y})$, and where the pair $\left(X_{i}, Y_{i}\right)$ is obtained from $\left(X_{i-1}, Y_{i-1}\right)$ by an elementary transformation for $i=1, \ldots, l$. Now suppose $\langle x, y ; \hat{U}(x, y), \ldots\rangle$ is a presentation of $\operatorname{sgp}\{\hat{X}, \hat{Y}\}$ associated with the generators $(\hat{X}, \hat{Y})$. Then a presentation $\left\langle x, y: U_{l-1}(x, y), \ldots\right\rangle$ for $\operatorname{sgp}\left\{X_{l-1}, Y_{l-1}\right\}$ associated with the generators $X_{l-1}, Y_{l-1}$ is derived as follows. If $(\hat{X}, \hat{Y})$ is obtained from $\left(X_{l-1}, Y_{l-1}\right)$ by simply interchanging $X_{l-1}$ and $Y_{l-1}$ then take $U_{l-1}(x, y)=\hat{U}(y, x), \ldots$; if $(\hat{X}, \hat{Y})=\left(X_{l-1}^{\varepsilon_{1}}, Y_{l-1}^{\varepsilon_{2}}\right)$ take $U_{l-1}(x, y)=\hat{U}\left(x^{\varepsilon_{1}}, y^{\varepsilon_{2}}\right), \ldots$; if $(\hat{X}, \hat{Y})$ is obtained from $\left(X_{l-1}, Y_{l-1}\right)$ by replacing $X_{l-1}$ by the $t$-reduced form of $X_{l-1} Y_{l-1}^{e}$ and leaving $Y_{l-1}$ fixed take $U_{l-1}(x, y)=\hat{U}\left(x y^{e}, y\right), \ldots$, and for other transformations as in (c) proceed similarly; if $(\hat{X}, \hat{Y})$ is obtained from $\left(X_{l-1}, Y_{l-1}\right)$ by a transformation as in (d), then take $U_{l-1}(x, y)=\hat{U}(x, y), \ldots$. Now using the presentation for $\operatorname{sgp}\left\{X_{l-1}, Y_{l-1}\right\}$ one obtains, in a similar manner, a presentation for $\operatorname{sgp}\left\{X_{l-2}, Y_{l-2}\right\}$, and so on, finally obtaining a presentation for $\operatorname{sgp}\{X, Y\}$ associated with the generators $X, Y$.

It will now be indicated how to obtain a presentation for $\operatorname{sgp}\{\hat{X}, \hat{Y}\}$ associated with the generators $\hat{X}, \hat{Y}$.

If (1) holds then all that is required is to know the orders of $\hat{X}$ and $\hat{Y}$. In this connection it is useful to recall (see [8, Theorem II]) that the only elements of finite order in $G$ are conjugates of elements of finite order in $H$.

If (2) holds then use the presentation $\langle x, y ; W(x, y)(W \in \mathbf{D}(\hat{X}, \hat{Y}))\rangle$.

(2) It, of course, follows from general subgroup theorems [2] that every two-generator subgroup of $G$ is an $H N N$ group. 
Suppose (3) holds. Then it follows from Lemma 3 of [5] that $r=1$, so that $\hat{X}=t h$ for some $t$-free word $h$, and $\hat{Y}=a^{m}$ for some integer $m$. To obtain a presentation for $\operatorname{sgp}\left\{t h, a^{m}\right\}$ consider the three generators $t h, a^{m}, h^{-1} b^{m} h$ $\left(=(t h)^{-1} a^{m}(t h)\right)$. Suppose the following equations hold:

$$
\begin{aligned}
& \operatorname{sgp}\left\{a^{m}, h^{-1} b^{m} h\right\} \cap \operatorname{sgp}\{a\}=\operatorname{sgp}\left\{a^{m}\right\} ; \\
& \operatorname{sgp}\left\{h a^{m} h^{-1}, b^{m}\right\} \cap \operatorname{sgp}\{b\}=\operatorname{sgp}\left\{b^{m}\right\} .
\end{aligned}
$$

Then it follows that:

a set of defining relations between the generators th, $a^{m}, h^{-1} b^{m} h$ is obtained by finding a set of defining relations between the generators $a^{m}, h^{-1} b^{m} h$ and then adding the additional relation $(t h)^{-1} a^{m}(t h)=h^{-1} b^{m} h$.

To prove this it must be shown that every word $W$ in $t h, a^{m}, h^{-1} b^{m} h$ which is equal to 1 is a consequence of the relations as described. The proof is by induction on the number of occurrences of $t h$ in $W$. If there are none then the fact that $W$ is equal to 1 is a consequence of the defining relations between $a^{m}$ and $h^{-1} b^{m} h$. Suppose now that $W$ involves $t h$ and that $W=1$. Then it follows from Britton's Lemma that $W$ has a subword $t^{*} k t^{-\ell}$ where $k$ is a word in $a$ and $b$ which defines an element of $\operatorname{sgp}\{a\}($ if $\varepsilon=-1$ ) or $\operatorname{sgp}\{b\}$ (if $\varepsilon=1)$. Consequently, either $W$ has a subword $(t h)^{-1} w(t h)$ where $w$ is a word in $a^{m}, h^{-1} b^{m} h$ which is equal to a power of $a$, or $W$ has a subword $(t h) w(t h)^{-1}$ where $w$ is a word in $a^{m}, h^{-1} b^{m} h$ which is equal to a power of $b$. If the former case occurs then it follows from (4) that $w$ is equal to a power $\left(a^{m}\right)^{p}$ of $a^{m}$, and this equality is, of course, a consequence of the defining relations between $a^{m}$ and $h^{-1} b^{m} h$. Now the relation $(t h)^{-1} a^{m}(t h)=h^{-1} b^{m} h$ implies that $(t h)^{-1}\left(a^{m}\right)^{p}(t h)=\left(h^{-1} b^{m} h\right)^{p}$, and replacing the subword $(t h)^{-1} w(t h)$ of $W$ by $\left(h^{-1} b^{m} h\right)^{p}$ gives a word $W^{\prime}$ with less occurrences of $t h$, and so the inductive hypothesis applies to $W^{\prime}$. If the latter case occurs then a similar argument can be employed, making use of (5).

It now follows from $(\dagger)$ that $\left\langle x, y ; W\left(x, y^{-1} x y\right) \quad(W(x, y) \in\right.$ $\left.\left.\mathbf{D}\left(a^{m}, h^{-1} b^{m} h\right)\right)\right\rangle$ is a presentation for $\operatorname{sgp}\left\{t h, a^{m}\right\}$ associated with the generators $t h, a^{m}$.

A few applications of the general results described above will now be given.

(A) Let $H=\left\langle a, b ; R_{1}, R_{2}, \ldots\right\rangle$. Assume that $\operatorname{sgp}\{a\}$ and $\operatorname{sgp}\{b\}$ are malnormal in $H$. Assume also that for all words $h$ in $a$ and $b$ and all integers $m$, (4) and (5) hold, and that furthermore, $\operatorname{sgp}\left\{a^{m}, h^{-1} b^{m} h\right\}$ is free of rank 2 untess either $m=0$, or $|m|=1$ and $h=b^{\kappa} a^{\rho}$ for suitable integers $\kappa, \rho$. Let $G=$ $\left\langle a, b, t ; R_{1}, R_{2}, \ldots, t^{-1} a t=b\right\rangle$. Then every two-generator group properly contained in $G$ is isomorphic either to a subgroup of $H$ or a free product of cyclic groups. 
Although the conditions imposed on $H$ in (A) are quite severe it can be shown that they are satisfied for various groups which are combinatorially similar to the free group of rank 2. (See [5, \$§3.1, 3.2.].)

The conditions of (A) are satisfied in particular if $H=\left\langle a, b ;\left(a^{r} b\right)^{l}\right.$, $\left.\left(a^{r+1} b\right)^{n}\right\rangle(|l|,|n| \neq 1$; if $l \neq 0$ then $r \neq 0$; if $n \neq 0$ then $r \neq-1)$. Since $H$ is a free product of cyclic groups every subgroup of $H$ is also a free product of cyclic groups $[3$, p. 243]. This gives the first part of the next result.

(B) Let $G=\left\langle a, t ;\left(a^{r} t^{-1} a t\right)^{l},\left(a^{r+1} t^{-1} a t\right)^{n}\right\rangle(|l|,|n| \neq 1$; if $l \neq 0$ then $r \neq 0$; if $n \neq 0$ then $r \neq-1)$. Every two-generator group properly contained in $G$ is a free product of cyclic groups. Moreover, there is an algorithm which, given any pair of words $(u, v)$ in $a, t$ either enables one to deduce that $u$ and $v$ generate $G$, or produces a pair of associated primitives $P, Q$ in the free group on $x, y$ and $a$ pair of integers $p, q$ such that $\left\langle x, y ; P^{p}, Q^{q}\right\rangle$ is a presentation of $\operatorname{sgp}\{u, v\}$ associated with the pair $u, v$.

To obtain the algorithm described in the second part of (B) one uses the fact that in the group $H=\left\langle a, b ;\left(a^{r} b\right)^{l},\left(a^{r+1} b\right)^{n}\right\rangle$ there is an effective procedure for calculating for any pair of words $h_{1}, h_{2}$ in $a, b$ a pair of associated primitives $S, T$ in the free group on $x, y$ and a pair of integers $\alpha, \beta$ such that $\left\langle x, y ; S^{\alpha}, T^{\beta}\right\rangle$ is a presentation of $\operatorname{sgp}\left\{h_{1}, h_{2}\right\}$ associated with $h_{1}, h_{2}$.

By making use of (B) it is possible to confirm a conjecture of $\mathbf{B}$. $\mathbf{H}$. Neumann. Several years ago K. Honda [1] proved the following theorem: if $G$ is a finite group and $g$ is a commutator in $G$ then every generator of $\operatorname{sgp}\{g\}$ is a commutator. Neumann has conjectured that this result should no longer be true in general for infinite groups, and Jim Wiegold has suggested that an example to verify this might be found by considering the group $G=$ $\left\langle a, t ;[a, t]^{5}\right\rangle$ and showing that $[a, t]^{2}$ is not a commutator. Wiegold's suggestion is taken up in the next result.

(C) Let $G=\left\langle a, t ;[a, t]^{n}\right\rangle$ with $n>1$. Then $[a, t]^{m}(0<m<n)$ is a commutator in $G$ if and only if $m=1, n-1$.

To prove this, suppose $[u, v]=[a, t]^{m}$. Then $u$ and $v$ must generate $G$. For otherwise $\operatorname{sgp}\{u, v\}$ would be a free product of two cyclic groups by (B) and so the derived group of $\operatorname{sgp}\{u, v\}$ would be free and therefore torsion-free (see [3, p. 113]). Now $G$ has one Nielsen equivalence class of generating pairs (see [7, Principal Lemma] $\left.{ }^{3}\right)$ ), and so it follows from Theorem 3.9 of [3] that $[u, v]=T^{-1}[a, t]^{e} T$ for some element $T$ of $G$. It now follows from general results concerning one-relator groups with torsion that $T \in \operatorname{sgp}\{[a, t]\}$ and $m \equiv \varepsilon \bmod n$. However, in the present special case this can be established directly as follows. Consider the $H N N$ presentation of $G$ with base $H=$

$\left({ }^{3}\right)$ For a simpler derivation of this fact in the present special case, see [4, Theorem 1]. 
$\left\langle a, b ;\left(a^{-1} b\right)^{n}\right\rangle$, stable letter $t$, and associated subgroups $\operatorname{sgp}\{a\}, \operatorname{sgp}\{b\}$, and assume without loss of generality that $T$ is a $t$-reduced word. Since $T^{-1}\left(a^{-1} b\right)^{e} T=\left(a^{-1} b\right)^{m}, T$ must be $t$-free, for $a^{-1} b$ is not conjugate in $H$ to a power of either $a$ or $b\left(a^{-1} b\right.$ has finite order whereas $a$ and $b$ have infinite order!). Thus the equation $T^{-1}\left(a^{-1} b\right)^{e} T=\left(a^{-1} b\right)^{m}$ takes place in the free product $H$, and it is not difficult to verify using the theory of free products that $T$ is a power of $a^{-1} b$ and $m \equiv \varepsilon \bmod n$.

The proof of the Main Theorem will now be given. The proof is by induction on $L(R)$.

If $L(R)=0,1$, or more generally if $R$ involves at most one generator, then the result follows using the theory of free products of cyclic groups.

Suppose $R$ involves at least two generators, say $a, t$.

Case A. Either $\sigma_{a}(R)=0$ or $\sigma_{t}(R)=0$.

Assume for definiteness that $\sigma_{t}(R)=0$. Calculations will be done with reference to the $H N N$ presentation of $G$ with stable letter $t$ and fixed generator $a$. The base $H$ of $G$ is a one-relator group with relator $P^{n}$, where $L(P)<L(R)$. It therefore follows from Theorem 1 that a description of the two-generator subgroups of $G$ will be completed once a description of the two-generator subgroups generated by a pair of the form $\left(t h_{1} \cdots t h_{r}, k\right)$-where $r>0$, the $h_{i}$ are $t$-free, $k$ is a nontrivial element of $K_{-1},\left(t h_{1} \cdots t h_{r}\right)^{-1} k\left(t h_{1} \cdots t h_{r}\right) t$-reduces to a $t$-free word-is obtained.

It can be assumed without loss of generality that $k$ is a nonempty cyclically reduced word in the generators of $K_{-1}$. For suppose $k \equiv z^{-1} \overline{k z}$ where $\bar{k}$ is cyclically reduced. Let $z_{1}$ be the $t$-reduced form of $t^{-1} z t$, and consider instead of the pair $\left(t h_{1} \cdots t h_{r}, k\right)$ the pair $\left(t z_{1} h_{1} \cdots t h_{r} z^{-1}, \bar{k}\right)$.

Now since $\left(t h_{1} \cdots t h_{r}\right)^{-1} k\left(t h_{1} \cdots t h_{r}\right) t$-reduces to a $t$-free word, it follows from Lemma 5 of [7] that $t^{-r}\left(t h_{1} \cdots t h_{r}\right) t$-reduces to a $t$-free word $h$, and it therefore suffices to consider $\operatorname{sgp}\left\{t^{r} h, k\right\}$. If $h$ and $k$ both belong to $\operatorname{sgp}\left\{b_{i}(i \in \mathbf{Z}), c_{i}(i \in \mathbf{Z}), \ldots\right\}$ then $\operatorname{sgp}\left\{t^{\prime} h, k\right\}$ is contained in the free group $\operatorname{sgp}\{t, b, c, \ldots\}$, and is therefore itself free.

Suppose that not both of $h, k$ belong to $\operatorname{sgp}\left\{b_{i}(i \in \mathbf{Z}), c_{i}(i \in \mathbf{Z}), \ldots\right\}$. As in (A) of [7], up to conjugation by a power of $t$ it can be assumed that $h k h^{-1} \notin K_{1}$. Let $k^{(0)}, \ldots, k^{(\lambda)}$ be the standard $H$-elements associated with $\left(t^{r} h, k\right)$. Then $\operatorname{sgp}\left\{t^{r} h, k\right\}$ is, of course, generated by the elements $t^{t} h, k^{(0)}, \ldots, k^{(\lambda)}$. Now it turns out that a result analogous to $(\dagger)$ holds. In fact:

a set of defining relations between the generators $t^{r} h, k^{(0)}, \ldots, k^{(\lambda)}$ is obtained by finding a set of defining relations between the generators $k^{(0)}, \ldots, k^{(\lambda)}$ and then adding the additional relations $\left(t^{r} h\right)^{-1} k^{(i)}\left(t^{r} h\right)=k^{(i+1)}$ for $i=$ $0, \ldots, \lambda-1$. 
(See Proposition 1, §3.)

Now assume first that $\lambda>1$ so that $h \in K_{-1}$. If $h k^{(\lambda)} h^{-1} \in K_{-1}$ then $h k^{(0)} h^{-1}, \ldots, h k^{(\lambda)} h^{-1}$ freely generate a subgroup of $K_{-1}$, and so it follows from ( $(\dagger)$ that $\operatorname{sgp}\left\{t^{r} h, k\right\}$ is free of rank 2. On the other hand if $h k^{(\lambda)} h^{-1} \in$ $K_{1} \backslash K_{-1}$ then $\left(h k^{(0)} h^{-1}, \ldots, h k^{(\lambda)} h^{-1}\right)$ is $\left(a_{0}, a_{M}\right)$-admissible, and so it follows from Theorem 1 of [6] that either $h k^{(0)} h^{-1}, \ldots, h k^{(\lambda)} h^{-1}$ freely generate a subgroup of $H$, or some conjugate of the relator $P^{n}$ of $H$ is freely expressible as a word $Q^{n}\left(h k^{(0)} h^{-1}, \ldots, h k^{(\lambda)} h^{-1}\right)$ in $h k^{(0)} h^{-1}, \ldots, h k^{(\lambda)} h^{-1}$ and every relation between $h k^{(0)} h^{-1}, \ldots, h k^{(\lambda)} h^{-1}$ is a consequence of the relation $Q^{n}=1$. If the former situation occurs then it follows from ( $\dagger \dagger$ ) that $\operatorname{sgp}\left\{t^{r} h, k\right\}$ is free of rank 2 , while if the latter situation occurs it follows from $(\dagger \dagger)$ that every relation between $t^{r} h$ and $k$ is a consequence of the single relation $Q^{n}\left(k, \ldots,\left(t^{r} h\right)^{-\lambda} k\left(t^{r} h\right)^{\lambda}\right)=1$.

Now suppose that $\lambda=1$. Then it follows from the inductive hypothesis that $\operatorname{sgp}\left\{k^{(0)}, k^{(1)}\right\}$ is either a free product of cyclic groups or a one-relator group with torsion, and naturally one would hope to use this fact together with ( $f \dagger)$ to deduce that $\operatorname{sgp}\left\{t^{r} h, k\right\}$ is also either a free product of cyclic groups or a one-relator group with torsion. However, suppose that $\operatorname{sgp}\left\{k^{(0)}, k^{(1)}\right\}$ were a free product of two finite cyclic groups. Then by the Grushko-Neumann Theorem there would be a pair of associated primitives $S(x, y), T(x, y)$ in the free group on $x, y$ and a pair of integers $\alpha, \beta$ each greater than 1 such that every relation between $k^{(0)}$ and $k^{(1)}$ would be a consequence of the relations

$$
S^{\alpha}\left(k^{(0)}, k^{(1)}\right)=1, \quad T^{\beta}\left(k^{(0)}, k^{(1)}\right)=1 .
$$

It would then follow from ( $\left.\dagger^{\dagger}\right)$ that every relation between $t^{r} h$ and $k$ would be a consequence of the relations

$$
\begin{aligned}
& S^{\alpha}\left(k,\left(t^{r} h\right)^{-1} k\left(t^{r} h\right)\right)=1, \\
& T^{\beta}\left(k,\left(t^{r} h\right)^{-1} k\left(t^{r} h\right)\right)=1,
\end{aligned}
$$

and it can be deduced from this that $\operatorname{sgp}\left\{t^{r} h, k\right\}$ is not a free product of cyclic groups or a one-relator group with torsion. It therefore becomes necessary to show that $\operatorname{sgp}\left\{k^{(0)}, k^{(1)}\right\}$ is not a free product of two finite cyclic groups. This is immediate if $k^{(1)} \in K_{-1}$, for then $k^{(0)}$ and $k^{(1)}$ freely generate a subgroup of the free group $K_{-1}$. If $k^{(1)} \notin K_{-1}$ then the following result applies.

Let $B=\left\langle x_{j}(j \in J) ; V^{m}\right\rangle$ with $m>1$, and for $j \in J$ define $L_{j}$ to be the subgroup of $B$ generated by the generators of $B$ other than $x_{j}$. Then it will be shown in $\$ 3$ that $B$ (or more precisely this presentation of $B$ ) has the following property: for each $j \in J$, if $u \in L_{j}(u \neq 1)$, and $v \notin L_{j}$, then every relation between $u$ and $v$ is a consequence of a single relation $Q^{\prime}(u, v)=1$, $l>1$ (where $Q$ may be freely equal to the empty word). This property will be 
referred to in the sequel as property-SR( $\left.{ }^{4}\right)$. It is worth remarking that the proof of this result gives a method for calculating for any pair $u, v$ as above a defining relation $Q^{l}(u, v)=1$ between $u$ and $v$, and in the case when $B$ is countably generated this method can be developed into an algorithm.

It now follows that every relation between $k^{(0)}$ and $k^{(1)}$ is a consequence of a single relation $Q^{\prime}\left(k^{(0)}, k^{(1)}\right)=1$ with $l>1$ (even, as remarked above, if $k^{(1)} \in K_{-1}$ ), and so, by $(\dagger)$, every relation between $t^{\prime} h$ and $k$ is a consequence of the single relation $Q^{\prime}\left(k,\left(t^{r} h\right)^{-1} k\left(t^{r} h\right)\right)=1$.

This completes the argument for Case $A$.

Case B. $\sigma_{a}(R) \neq 0$ and $\sigma_{t}(R) \neq 0$.

Let $\alpha=\sigma_{a}(R)$ and $\tau=\sigma_{t}(R)$, and let $\bar{G}=\left\langle\bar{t}, \bar{a}, b, c, \ldots ; \bar{R}^{n}\right\rangle$, where $\bar{R}$ is the word obtained from $R$ by replacing $t$ by $t^{\alpha}$ and $a$ by $\bar{a} \bar{t}^{-\tau}$ and then cyclically reducing. Then $G$ is embedded into $\bar{G}$ by the mapping.

$$
t \mapsto \bar{t}^{\alpha}, \quad a \mapsto \bar{a} \bar{t}^{-\tau}, \quad b \mapsto b, \quad c \mapsto c, \ldots
$$

Now $\sigma_{i}(\bar{R})=0$, and so one can consider the $H N N$ presentation of $\vec{G}$ with stable letter $\bar{t}$ and fixed generator $\bar{a}$. The base is then another one-relator group with relator $\bar{P}^{n}$ and the length of $\bar{P}$ is less than $L(R)$. One can therefore use an argument similar to that used in Case $A$ to describe the two-generator subgroups of $\bar{G}$, and so, in particular, the two-generator subgroups of $G$.

2. Proof of Theorem 1. Only the first part of Theorem 1 will be proved explicitly; the reader will be able to obtain from this the proof needed for the second part. The proof follows fairly closely the proof of Theorem 6 of [5], and certain parts of the proof which are similar to parts of the proof of Theorem 6 of [5] will be omitted.

Let $(X, Y)$ be a pair of $t$-reduced words in the generators of $G$, and obtain from $(X, Y)$ the pair $(\tilde{X}, \tilde{Y})$ as in Theorem 5 of [5]. If $|\tilde{Y}|>0$ then $\operatorname{sgp}\{\tilde{X}, \tilde{Y}\}$ is free of rank 2 and in this case it suffices to take $(\hat{X}, \hat{Y})$ to be the pair $(\dot{\tilde{X}}, \tilde{Y})$.

Suppose $|\tilde{Y}|=0$. Let $T$ be the initial segment of $\tilde{X}$ such that the $t$-reduced form of $T^{-1} \tilde{X} T$ is the cyclically $t$-reduced form $U$ of $\tilde{X}$. By Lemma 6 of [5] there is a $t$-free word $h$ and a terminal segment $T_{2}$ of $T$, which is either empty or has $t$-length greater than zero, such that the $t$-reduced form of $T^{-1} \tilde{Y} T$ is $T_{2}^{-1} h T_{2}$.

If $\left|T_{2}\right|>0$ then $\operatorname{sgp}\left\{U, T_{2}^{-1} h T_{2}\right\}=\operatorname{sgp}\{U\} * \operatorname{sgp}\left\{T_{2}^{-1} h T_{2}\right\}$ (see [5, p. 355]), so in this case one can take $(\hat{X}, \hat{Y})$ to be $\left(U, T_{2}^{-1} h T_{2}\right)$.

Suppose $T_{2}$ is empty. If $U$ is $t$-free then take $(\hat{X}, \hat{Y})$ to be $(U, h)$. Assume from now on that $U$ involves $t$.

(4) Single relation. 
Case A. For each integer $n$ with $h^{n} \neq 1, U h^{n} U, U^{-1} h^{n} U, U h^{n} U^{-1}$ are $t$-reduced.

Then $\operatorname{sgp}\{U, h\}=\operatorname{sgp}\{U\} * \operatorname{sgp}\{h\}$. Take $(\hat{X}, \hat{Y})$ to be the pair $(U, h)$.

Case B. For some integer $n_{0}$ with $h^{n_{0}} \neq 1, U h^{n_{0}} U$ is not $t$-reduced.

Then $U^{-1} h^{n} U$ and $U h^{n} U^{-1}$ are $t$-reduced for all integers $n$ for which $h^{n} \neq 1$ (see [5, p. 355]).

Suppose that for every integer $n$, at most $[(|U|-1) / 2] t$-symbols are removed from each copy of $U$ in $t$-reducing $U h^{n} U$. Then it is not difficult to see $\left[5\right.$, p. 356] that after $t$-reducing a word $U^{m_{1}} h^{n_{1}} U^{m_{2}} h^{n_{2}} \ldots U^{m_{1}} h^{m_{1}}(l>$ $0, m_{i} \neq 0, h^{m_{1}} \neq 1$ for $\left.i=1, \ldots, l\right)$ at least one $t$-symbol from each occurrence of $U$ remains, so that $\operatorname{sgp}\{U, h\}=\operatorname{sgp}\{U\} * \operatorname{sgp}\{h\}$. It thus suffices to take $(\hat{X}, \hat{Y})$ to be the pair $(U, h)$.

Now suppose that $U \equiv P Q$ where $Q h^{n} P$ t-reduces to a $t$-free word $u$ for some integer $n$. Then transform $(U, h)$ to $\left(u, P^{-1} h P\right)$. Now since $P^{-1} h P$ and $P u P^{-1}$ are $t$-reduced it follows, using Lemma 9 of [5], that $\operatorname{sgp}\left\{u, P^{-1} h P\right\}=$ $\operatorname{sgp}\{u\} * \operatorname{sgp}\left\{P^{-1} h P\right\}$. Take $(\hat{X}, \hat{Y})$ to be the pair $\left(u, P^{-1} h P\right)$.

Case C. For some integer $n_{0}$ with $h^{n_{0}} \neq 1, U^{-1} h^{n_{0}} U$ is not $t$-reduced.

Then $U^{-1} h^{n} U$ is not $t$-reduced for all integers $n$ with $h^{n} \neq 1$, and $U h^{n} U$ is $t$-reduced for all integers $n$ (see [5, p. 356]).

Suppose $p t$-symbols from $U$ are used up in $t$-reducing $U^{-1} h U$. Let $P$ be the initial segment of $U$ up to and including the $p$ th $t$-symbol of $U$, and let $U_{1}$ be the rest of $U$. Suppose that $q t$-symbols from $U_{1}$ are used up in $t$-reducing $U_{1} h U_{1}^{-1}$ and let $Q^{-1}$ be the initial segment of $U_{1}^{-1}$ up to and including the $q$ th $t$-symbol (thus $Q$ is empty if $q=0$ ). Then $U \equiv P \bar{U} Q$. Now it is not difficult to show that if $\bar{U}$ is not $t$-free then $\operatorname{sgp}\{U, h\}=$ $\operatorname{sgp}\{U\} * \operatorname{sgp}\{h\}$ (see [5, pp. 356-357]), and so in this situation one can take $(\hat{X}, \hat{Y})$ to be the pair $(U, h)$.

If $\bar{U}$ is $t$-free then transform the pair $(P \bar{U} Q, h)$ to $(Q P \bar{U}, v)$, where $v$ is the $t$-reduced form of $Q h Q^{-1}$. Let $Z$ denote the word $Q P \bar{U}$. Then $Z^{-1} v Z$ $t$-reduces to a $t$-free word. This implies that all the exponents to which $t$ occurs in $Z$ have the same sign. For suppose by way of contradiction that $Z \equiv S z V$ where $S$ has last symbol $t^{-\varepsilon}, V$ has first symbol $t^{\ell}$, and $z$ is $t$-free. Now $S^{-1} v S$-reduces to give a nontrivial element $k$ of $K_{-e}$, and since $V^{-1} z^{-1} k z V$ is not $t$-reduced it follows that $z^{-1} k z \in K_{-e}$, which implies $z \in K_{-\varepsilon}$ by malnormality. But this contradicts the fact that $Z$ is $t$-reduced.

It has been shown that $Z$ has the form $z_{0} t^{2} z_{1} t^{2} z_{2} \cdots t^{2} z_{r}$ where the $z_{i}$ are $t$-free. If $\varepsilon=1$ then take $(\hat{X}, \hat{Y})$ to be the pair $\left(t z_{1} t z_{2} \cdots t z_{r} z_{0}, z_{0}^{-1} v z_{0}\right)$. If $\varepsilon=-1$ then take $(\hat{X}, \hat{Y})$ to be the pair $\left(t z_{r-1}^{-1} \cdots t z_{1}^{-1} t z_{0}^{-1} z_{r}^{-1}, z_{r} w z_{r}^{-1}\right)$, where $w$ is the $t$-reduced form of $Z^{-1} v Z$.

Case D. For some integer $n_{0}$ with $h^{n_{0}} \neq 1$ in $G, U h^{n_{0}} U^{-1}$ is not $t$-reduced.

An argument similar to that used in Case $C$ above (replace $U$ by $U^{-1}$ ) applies. 
3. Property-SR. The aim of this section is to establish the following result.

Let $B=\left\langle x, y, b, \ldots ; R^{n}\right\rangle$ where $R$ is cyclically reduced and $\left.n\right\rangle 1$. Then $B$ has property-SR.

This result will be proved by induction on the length of $R$, making use of the fact that if $R$ involves at least two generators then $B$ can be embedded into an $H N N$ group whose base is a one-relator group, the relator $P^{n}$ of which has length less than $L\left(R^{n}\right)$. The following three results will therefore be useful. All of the results refer to a fixed but arbitrary $H N N$ group $G$ with the following structure. Let

$$
L=\left\langle a_{0}, \ldots, a_{N}, c_{i}(i \in \mathbf{Z}), d_{i}(i \in \mathbf{Z}), \ldots ; Q^{n}\right\rangle
$$

where $N>0, n>1$ and $Q$ is a cyclically reduced word which involves $a_{0}$ and $a_{N}$. Then $G$ is the $H N N$ group with base $L$, stable letter $t$, and associated subgroups

and

$$
A_{-1}=\operatorname{sgp}\left\{a_{0}, \ldots, a_{N-1}, c_{i}(i \in \mathbf{Z}), d_{i}(i \in \mathbf{Z}), \ldots\right\}
$$

$$
A_{1}=\operatorname{sgp}\left\{a_{1}, \ldots, a_{N}, c_{i}(i \in \mathbf{Z}), d_{i}(i \in \mathbf{Z}), \ldots\right\} .
$$

The action of $t$ is given by

$$
\begin{gathered}
t^{-1} a_{i} t=a_{i+1} \quad \text { for } i=0, \ldots, N-1, \\
t^{-1} b_{i} t=b_{i+1}, \quad t^{-1} c_{i} t=c_{i+1}, \ldots \quad(i \in \mathbf{Z}) .
\end{gathered}
$$

Proposition 1. Let $p$ be a positive integer, let $k$ be a nonempty cyclically reduced word in the generators of $A_{-1}$, and let $h$ be a $t$-free word. Assume that $h k^{-1} \notin A_{1}$ and let $k^{(0)}, \ldots, k^{(\lambda)}$ be the standard L-elements. Then a set of defining relations between the generators $t^{p} h, k^{(0)}, \ldots, k^{(\lambda)}$ of $\operatorname{sgp}\left\{t^{p} h, k\right\}$ is obtained by finding a set of defining relations between $k^{(0)}, \ldots, k^{(\lambda)}$ and then adding the relations $\left(t^{p} h\right)^{-1} k^{(i)}\left(t^{p} h\right)=k^{(i+1)}, i=0, \ldots, \lambda-1$.

This follows from Proposition 1 of [7] and its Corollary, together with the fact that $L$ has property-I by Theorem 4 of [7].

Note that Proposition 1 was used above to obtain ( $\dagger \dagger$ ).

Proposition 2. Let $u$ be a cyclically reduced word in $a_{0}, c_{0}, d_{0}, \ldots$ which involves $a_{0}$, and let $v$ be an element of $G$ which does not belong to $\operatorname{sgp}\left\{a_{0}, c_{0}, d_{0}, \ldots\right\}$. Suppose that $L$ has property-SR. Then every relation between $u$ and $v$ is a consequence of a single relation $T^{l}(u, v)=1$, with $l>1$.

This result is a corollary of the proof of Proposition 2 of [7]. Following through the proof of that proposition it can be seen that either $\operatorname{sgp}\{u, v\}$ is a free product of nontrivial cyclic groups, one of infinite order, or there are integers $m, r, \varepsilon$ with $0<r<N$ such that $t^{-r}\left(v u^{m}\right)^{\varepsilon} t^{r}=t^{p} h$, where $0<p, h$ is $t$-free, $\left(t^{p} h\right)^{-1} u_{r}\left(t^{p} h\right) \in L, h u_{r} h^{-1} \notin A_{1}$ if $p>0$ (here $u_{r}$ is the word 
obtained from $u$ by replacing $a_{0}$ by $a_{r}, c_{0}$ by $c_{r}, d_{0}$ by $d_{r}, \ldots$ ). In the former case it follows from the Grushko-Neumann Theorem that every relation between $u$ and $v$ is a consequence of a single relation $T^{l}(u, v)=1$ with $l>1$. In the latter case it suffices to show that every relation between $t^{p} h$ and $u_{r}$ is a consequence of a single relation $S^{q}\left(t^{p} h, u_{r}\right)=1$ with $q>1$. If $p=0$ this follows from the fact that $L$ has property-SR (note that $h \notin$ $\left.\operatorname{sgp}\left\{a_{r}, c_{r}, d_{r}, \ldots\right\}\right)$.

Suppose $p>0$, and let $u_{r}^{(0)}, \ldots, u_{r}^{(\lambda)}$ be the standard $L$-elements. Then every relation between $u_{r}^{(0)}, \ldots, u_{r}^{(\lambda)}$ is a consequence of a single relation $V^{q}\left(u_{r}^{(0)}, \ldots, u_{r}^{(\lambda)}\right)=1$, with $q>1$. This follows from the fact that $L$ has property-SR in the case when $\lambda=1$. On the other hand suppose $\lambda>1$, so that $h \in A_{-1}$. If $u_{r}^{(\lambda)} \notin A_{-1}$ then $\left(h u_{r}^{(0)} h^{-1}, \ldots, h u_{r}^{(\lambda)} h^{-1}\right)$ is $\left(a_{0}, a_{N}\right)$-admissible and so Theorem 1 of [6] applies, whereas if $u_{r}^{(\lambda)} \in A_{-1}$ then $u_{r}^{(0)}, \ldots, u_{r}^{(\lambda)}$ freely generate a subgroup of $A_{-1}$. It now follows from Proposition 1 that every relation between $t^{p h}$ and $u_{r}$ is a consequence of the single relation $V^{q}\left(u_{r}, \ldots,\left(t^{p} h\right)^{-\lambda} u_{r}\left(t^{p} h\right)^{\lambda}\right)=1$.

Proposition 3. Let $u$ be a nonempty freely reduced word in $t, c_{0}, d_{0}, \ldots$, and let $v$ be an element of $G$ which does not belong to $\operatorname{sgp}\left\{t, c_{0}, d_{0}, \ldots\right\}$. Suppose $L$ has property-SR. Then every relation between $u$ and $v$ is $a$ consequence of a single relation $T^{l}(u, v)=1$ with $l>1$.

Conjugating $u$ and $v$ by an element of $\operatorname{sgp}\left\{t, c_{0}, d_{0}, \ldots\right\}$ if necessary, it can be assumed without loss of generality that $u$ is a nonempty cyclically reduced word in $t, c_{0}, d_{0}, \ldots$.

Proposition 3 is a corollary of the proof of Proposition 3 of [7]. As in the proof of that proposition obtain from $(u, v)$ the pair $\left(t^{m} k, t^{p} z\right)$. Notice that if $m=0$ then $k$ is a nonempty cyclically reduced word in $c_{i}(i \in \mathbf{Z}), d_{i}(i \in$ Z), ... Notice also that there are integers, $q, \beta$, $\omega$ such that

$$
t^{m} k=t^{-q u t^{q}}, \quad t^{p} z=t^{-q_{u} \beta_{v u^{\omega}} t^{q},}
$$

so that in order to show that every relation between $u$ and $v$ is a consequence of a single relation $T^{l}(u, v)=1(l>1)$, it suffices to show that every relation between $t^{m} k$ and $t^{p_{Z}}$ is a consequence of a single relation $U^{l}\left(t^{m} k, t^{p_{z}}\right)=1$.

Follow through the various cases in the proof of Proposition 3 of [7]. Cases 1, 2, 3 and Subcases 4.2, 4.4 are straightforward. For Subcase 4.1 the result follows from the fact that $L$ has property-SR. Consider Subcase 4.3. One has $\left(t^{p}, k\right)$ with $p>0$, and there are just two standard $L$-elements $k^{(0)}, k^{(1)}$, where $k^{(0)} \in A_{-1}, k^{(1)} \notin A_{-1}, k^{(0)} \neq 1$. Since $L$ has property-SR, every relation between $k^{(0)}$ and $k^{(1)}$ is a consequence of a single relation $V^{\prime}\left(k^{(0)}, k^{(1)}\right)=1$, where $l>1$. Consequently, by Proposition 1 above, every relation between $t^{p_{z}}$ and $k$ is a consequence of the relation $V^{l}\left(k,\left(t^{p} z\right)^{-1} k\left(t^{p} z\right)\right)=1$. 
It will now be established that if $B=\left\langle x, y, b, \ldots ; R^{n}\right\rangle$, where $R$ is cyclically reduced and $n>1$, then $B$ has property-SR. As mentioned above the proof is by induction on $L(R)$.

If $L(R)=0$ then $B$ is freely generated by $x, y, b, \ldots$, and so every subgroup of $B$ is free. The result now follows easily.

Suppose that $L(R)>0$. Let $u$ be a freely reduced word in $y, b, \ldots$ with $u \neq 1$ and suppose $v \notin \operatorname{sgp}\{y, b, \ldots\}$. It will be shown that every relation between $u$ and $v$ is a consequence of a single relation $T^{l}(u, v)=1$ with $l>1$. It suffices to consider the situation where $u$ is a cyclically reduced word in $y, b, \ldots$

Case A. $x$ does not occur in $R$.

Then $B$ is the free product of the free group on $x$ and the one-relator group generated by the remaining generators. The result follows using the theory of free products.

Case B. No generator occurring in $u$ also occurs in $R$.

Let $F$ denote the free group on those generators which occur in $u$, and let $B^{\prime}$ be the one-relator group generated by the remaining generators of $B$. Then $B$ is the free product of $F$ and $B^{\prime}$, and $u \in F, v \notin F$. The result now follows using the theory of free products.

Case $C . x$ occurs in $R$ with zero-sum exponent, one of the generators occurs in both $u$ and $R$.

Suppose for definiteness that $y$ occurs in $u$ and $R$. Consider the $H N N$ presentation of $B$ with stable letter $x$ and fixed generator $y$. By the inductive hypothesis the base of $B$ has property-SR, so it follows from Proposition 2 that every relation between $u$ and $v$ is a consequence of a single relation $T^{l}(u, v)=1$ with $l>1$.

Case D. $x$ occurs in $R$, one of the generators which occurs in $u$ occurs in $R$ with zero-sum exponent.

Suppose $y$ occurs in $u$ and $R$ and $\sigma_{y}(R)=0$. Consider the $H N N$ presentation of $B$ with stable letter $y$ and fixed generator $x$. Then the base has property-SR by the inductive hypothesis, so the result follows from Proposition 3.

Case E. $x$ occurs in $R, \sigma_{x}(R) \neq 0$, one of the generators which occurs in $u$ occurs in $R$ with non-zero-sum exponent.

Suppose for definiteness that $y$ occurs in $u$ and $R$, and $\sigma_{y}(R) \neq 0$. Let $\alpha=\sigma_{x}(R)$ and $\beta=\sigma_{y}(R)$. Let $B_{1}=\left\langle t, a, b, \ldots ; R_{1}^{n}\right\rangle$, where $R_{1}$ is the word obtained from $R$ by replacing each occurrence of $x$ by at ${ }^{-\beta}$ and each occurrence of $y$ by $t^{\alpha}$, and then cyclically reducing. Then $B$ is embedded into $B_{1}$ by the homomorphism

$$
\Psi: x \mapsto a t^{-\beta}, \quad y \mapsto t^{\alpha}, \quad b \mapsto b, \ldots,
$$

and $\operatorname{sgp}\{t, b, \ldots\} \cap \Psi(B)=\operatorname{sgp}\left\{t^{\alpha}, b, \ldots\right\}$. 
Now $R_{1}$ certainly involves $a$ and, moreover, $\sigma_{t}\left(R_{1}\right)=0$. Thus one can consider the $H N N$ presentation of $B_{1}$ with stable letter $t$ and fixed generator $a$. The base of $B_{1}$ is another one-relator group, the relator of which has length less than $L\left(R^{n}\right)$. Consequently the base has property-SR by the inductive hypothesis, and so it follows from Proposition 3 that every relation between $\Psi(u)$ and $\Psi(v)$ is a consequence of a single relation $T^{l}(\Psi(u), \Psi(v))=1$ with $l>1$. Thus every relation between $u$ and $v$ is a consequence of $T^{l}(u, v)=1$.

\section{REFERENCES}

1. K. Honda, On commutators in finite groups, Comment. Math. Univ. St. Paul 2 (1953), 9-12. MR 15, 286.

2. A. Karrass and D. Solitar, Subgroups of $H N N$ groups and groups with one defining relation, Canad. J. Math. 23 (1971), 627-643. MR 46 \#260.

3. W. Magnus, A. Karrass and D. Solitar, Combinatorial group theory: Presentations of groups in terms of generators and relations, Pure and Appl. Math., Vol. 13, Interscience, New York, 1966. MR 34 \# 7617.

4. S. J. Pride, On the Nielsen equivalence of pairs of generators in certain HNN groups, Proc. Second. Internat. Conf. on Theory of Groups (Australian Nat. Univ., Canberra, 1973), Lecture Notes in Math., vol. 372, Springer-Verlag, Berlin and New York, 1974, pp. 580-588. MR 50 \#13288.

5. On the generation of one-relator groups, Trans. Amer. Math. Soc. 210 (1975), 331-364. MR 52 \#3341.

6. ___ Certain subgroups of certain one-relator groups, Math. Z. 146 (1976), 1-6.

7. The isomorphism problem for two-generator one-relator groups with torsion is solvable, Trans. Amer. Math. Soc. 227 (1977), 109-139.

8. P. E. Schupp, Some reflections on HNN extensions, Proc. Second Internat. Conf. on Theory of Groups (Australian Nat. Univ., Canberra, 1973), Lecture Notes in Math., vol. 372, SpringerVerlag, Berlin and New York, 1974, pp. 611-632. MR 50 \# 10085.

Department of Mathematics, The Open University, Milton Keynes, MK7 6AA, Bucks, ENGLAND 